MS28-P05

\section{Magnetic Raman scattering and symmetry analysis of complex-structure antiferromagnets $\mathrm{Ni}_{2} \mathrm{NbBO}_{6}$ and $\mathrm{Fe}_{3} \mathrm{BO}_{6}$}

Mikhail Prosnikov $^{1}$, Sergei Barilo ${ }^{2}$, Nadezhda Lyubochko ${ }^{2}$, Sergei Shiryaev $^{2}$,Alexander Smirnov ${ }^{1}$, Valery Davydov ${ }^{1}$, Roman Pisarev ${ }^{1}$

1. Ioffe Institute, Saint Petersburg, Russia

2. Institute of Solid State and Semiconductor Physics, Minsk, Belarus

email: yotungh@gmail.com

Isostructural orthorhombic crystals $\mathrm{Ni}_{2} \mathrm{NbBO}_{6}$ and $\mathrm{Fe}_{3} \mathrm{BO}_{6}$ belong to the Pnma (\#62) space group [1] and manifest 3D long-range AFM transitions at $\mathrm{T}_{\mathrm{N}}=23.5 \mathrm{~K}$ [2] and $\mathrm{T}_{\mathrm{N}}=508$ $\mathrm{K}$, respectively. Such difference in transition temperatures is mostly due to different connectivity; while in $\mathrm{Ni}_{2} \mathrm{NbBO}_{6}$ magnetic ions occupy only the general $8 d$ positions, in $\mathrm{Fe}_{3} \mathrm{BO}_{6}$ they are located in the $8 d$ and $4 c$ ones, which results in complitely distinct magnetic structures and superexchange paths. Other characteristic feature of $\mathrm{Fe}_{3} \mathrm{BO}_{6}$ is a presence of a weak ferromagnetic moment, which is spontaneously reoriented from [100] to [001] axis at $\mathrm{T}_{\mathrm{SO}}=415 \mathrm{~K}$. Thereby, these systems offer an unique playground to explore static/dynamic magnetism and symmetry requrements for the existance and orientation of the weak ferromagnetic moment.

We present the experimental results on the crystal growth and studies of both lattice and magnetic dynamics, and their interaction in single crystals of $\mathrm{Ni}_{2} \mathrm{NbBO}_{6}$ and $\mathrm{Fe}_{3} \mathrm{BO}_{6}$ with the use of the polarized Raman spectroscopy in a wide temperature range $10-550 \mathrm{~K}$. Nontrivial spin-phonon interaction with different signs of coupling constant depending on particular phonon mode was observed at $\mathrm{T}_{\mathrm{N}}$. In the AFM phases several magnetic modes of different nature were registered, including two-magnon ones. Methods of magnetic space groups and irreducible representations were used to determine the set of magnetic subgroups compatible with experimental observations. Appropriate Hamiltonians including several isotropic exchange and single-ion anisotropy constants were suggested for describing two-magnon excitations within the linear spin wave theory. Thus we were able to show that magnetic Raman scattering supported by symmetry analysis could be used for determinationof magnetic structures and exchange/anisotropy parameters [3].

References:

[1] Ansell, G. B. et al. (1982). Acta Cryst. B 38, 892-893.

[2] Rao, G. N. et al. (2015). Phys. Rev. B 91, 014423-5.

[3] Prosnikov M. A. et al. (2017). Phys. Rev. B 96, 014428-10.

Keywords: magnetic Raman scattering, symmetry analysis, magnetic space group symmetry and other topics

MS29-P01

\section{Towards objective crystallographic symmetry classifications of noisy $2 \mathrm{D}$ periodic images}

\author{
Peter Moeck ${ }^{1}$, Andrew Dempsey ${ }^{1}$ \\ 1. Nano-Crystallography Group, Department of Physics, Portland \\ State University, Portland, Oregon, United States of America \\ email:pmoeck@pdx.edu
}

Crystallographic symmetry classifications of noisy 2D periodic images are currently made on the basis of the three traditional plane symmetry deviation quantifiers of electron crystallography [1]. These quantifiers are, however, "pure distance measures" that are unable to deal with crystallographic supergroup-subgroup relationships and pseudo-symmetries in an objective manner [2]. A consequence of this is that the model with the lowest symmetry, i.e. the one which possesses the highest number of free parameters, fits noisy experimental data best. A version of Hamilton's well known R-factor ratio test [3] of mainstream 3D crystallography can be applied in principle, but is of limited utility because it is a null hypothesis test.

Objective crystallographic symmetry deviation quantifiers that properly account for crystallographic supergroup-subgroup relationships and pseudo-symmetries have recently been derived for noisy 2D periodic images on the basis of geometric Akaike Information Criteria (G-AICs) [2] and associated Akaike weights. (Akaike weights represent the probability that a certain crystallographic symmetry model within a disjoint or non-disjoint model set is the one that minimizes Kullback-Leibler information loss when it is used to represent full reality.) These quantifiers are demonstrated in this contribution on examples for the first time.

Openly accessible synthetic 2D periodic images (https:// nmevenkamp.github.io/UnitCellExtraction/) have been utilized for our objective crystallographic classifications with respect to their Bravais lattice types, Laue classes, and plane symmetry groups. The example images possess per design both genuine pseudo-symmetries and added Gaussian noise, which turned genuine symmetries into pseudo-symmetries of the second kind. Note that genuine symmetries constitute the symmetry group structure of the hypothetical noise-free version of an image, but are unavoidably disturbed by noise in any real world imaging process.

Genuine pseudo-symmetries and pseudo-symmetries of the second kind also cause problems in mainstream single crystal X-ray crystallography [2] so that the approach of this contribution should be generalized to the 3D case in order to achieve a larger impact. A few percent, i.e. tens of thousands, of the molecule and crystal structures in the major $3 \mathrm{D}$ crystallography databases have been mis-classified with respect to their crystallographic symmetry [2]. This is due to 